\title{
Comparison of Respiratory-Triggered (RT) 3D cine steady-state free precession cardiac mri with standard 2D cine imaging and magnetic resonance angiography in Congenital Heart Disease (CHD)
}

\author{
Lamya A Atweh ${ }^{1 *}$, Karen Lyons ${ }^{2}$, Ramkumar Krishnamurthy ${ }^{2}$, Amol Pednekar $^{3}$, Rajesh Krishnamurthy ${ }^{2}$
}

From 18th Annual SCMR Scientific Sessions

Nice, France. 4-7 February 2015

\section{Background}

This is a pilot study to evaluate the performance of whole-heart isotropic RT-3D cine SSFP sequence as an efficient single solution for assessment of morphology and function in CHD by comparing it to: 1 . Short axis (SA) 2D cine SSFP for LV volumetry and function 2. Multiplanar 2D cine SSFP for intracardiac morphology 3. Cine 2D SSFP and gadolinium (Gd)-enhanced 3D MRA for aortic root size 4. Gd-3D MRA for vessel diameter (VD) 5. Gd-3D MRA, and cine 2D SSFP for morphology of extracardiac vasculature 6. Gd-3D MRA and multiplanar cine 2D SSFP for overall diagnosis.

\section{Methods}

15 patients with CHD (8M, mean age 11.7 years), referred for clinic CMR (80\% sedated) underwent an additional RT 3D SSFP sequence (Table 1). LV volumetry was performed on the 3D and 2D cine SSFP SA datasets by the same reader on CMR42 software. VD measurements, image quality analysis, and pathologic findings were assessed on 3D, 2D SSFP and MRA datasets using Vitrea ${ }^{\mathrm{TM}}$ by another blinded reader. Pearson and Spearman rank correlation coefficients were calculated and paired $t$ tests performed to compare volumetry, imaging scores, maximal $\mathrm{VD}$, and diagnostic accuracy.

\section{Results}

1. Excellent correlation between LV volumetry from 3D SSFP and 2D SSFP ( $\mathrm{r}=0.91-0.99, \mathrm{p}<.0001)$. Only LVESV was significantly different (mean diff $-1.63 \mathrm{cc}, \mathrm{p} 0.03$ ). 2.

'Diagnostic Radiology, American University of Beirut Medical Center, Beirut, Lebanon

Full list of author information is available at the end of the article
Excellent correlation between aortic root measurements on 3D and 2D SSFP, and on 3D SSFP and MRA ( $r=0.98$, $0.96, \mathrm{p}<0.0001)$. Maximal intersinus root diameter was not significantly different. 3. Excellent correlation between VD on 3D SSFP and MRA ( $\mathrm{r}=0.96-0.99, \mathrm{p}<0.001)$. Only MPA diameter was significantly different (mean diff $-1.92 \mathrm{~mm}$, $\mathrm{p}$ 0.01). 4. Moderate performance for intracardiac morphology and pathology; 3D scores of aortic and tricuspid valves were lower than 2D (mean diff $-28.9 \%,-21.2 \%, p=0.003$, $0.005)$. Interslice alignment was better on $3 \mathrm{D}$ imaging (mean diff 8.9\%, $\mathrm{p}=0.04$ ). 5. There was no significant difference in the overall aorta and PA scores; but 3D cine SSFP scores were lower for evaluation of 1st and 2nd order vessel branches, and for AV separation (mean diff $-23 \%,-53 \%,-27 \%, p=0.025,<0.0001,0.01)$. 3D scores were also lower for the veins (mean diff $-25 \%,-29 \% \mathrm{p}=0.03$, $0.01) .6$. The overall diagnosis was different $(\mathrm{p}=0.02)$ in $5 /$ 15 cases ( 9 missed findings) on 3D imaging. 5/9 reflected valve pathology, 2 evaluation of 1st order vessels, 1 of aorta, and 1 of atrial septum. 4 of the missed findings were partly due to suppression of turbulence by gadofosveset. Comparison of 3D SSFP with conventional MR sequences are shown in a bar graph (Figure 1). Mean duration for 3D SSFP was $5.12 \pm 0.48$ min compared to $28.02 \pm 6.73 \mathrm{~min}$ for multiplanar 2D SSFP.

\section{Conclusions}

RT 3D cine SSFP can replace conventional MR sequences for LV volumetry and function, aortic root measurement, and vessel diameter. Improved hardware and software, and improved spatial resolution are needed before it can replace current sequences for evaluation of intracardiac morphology and extracardiac vasculature. 
Table 1 Respiratory Triggered 3-D Cine SSFP Technique and Grading Methodology

\begin{tabular}{|c|c|}
\hline Duration & 4.5-7 minutes \\
\hline Hardware & Phillips Achieva 1.5T magnet; 5-channel phased array coil; Max. parallel acceleration: 2 (2D); 2X1.5 (3D) \\
\hline Acquisition Parameters & $\begin{array}{l}\text { Axial or sagittal acquisition; respiratory triggered, retrospective EKG gated, TR/TE/ } \alpha=3 / 1.5 / 60 ; \text { voxel size 1.5-2.5; } \\
\qquad \text { SENSE 2.6-3.6; mean temporal resolution = } 35 \mathrm{~ms}\end{array}$ \\
\hline $\begin{array}{l}\text { Imaging Targets \& Grading } \\
\text { Methodology }\end{array}$ & 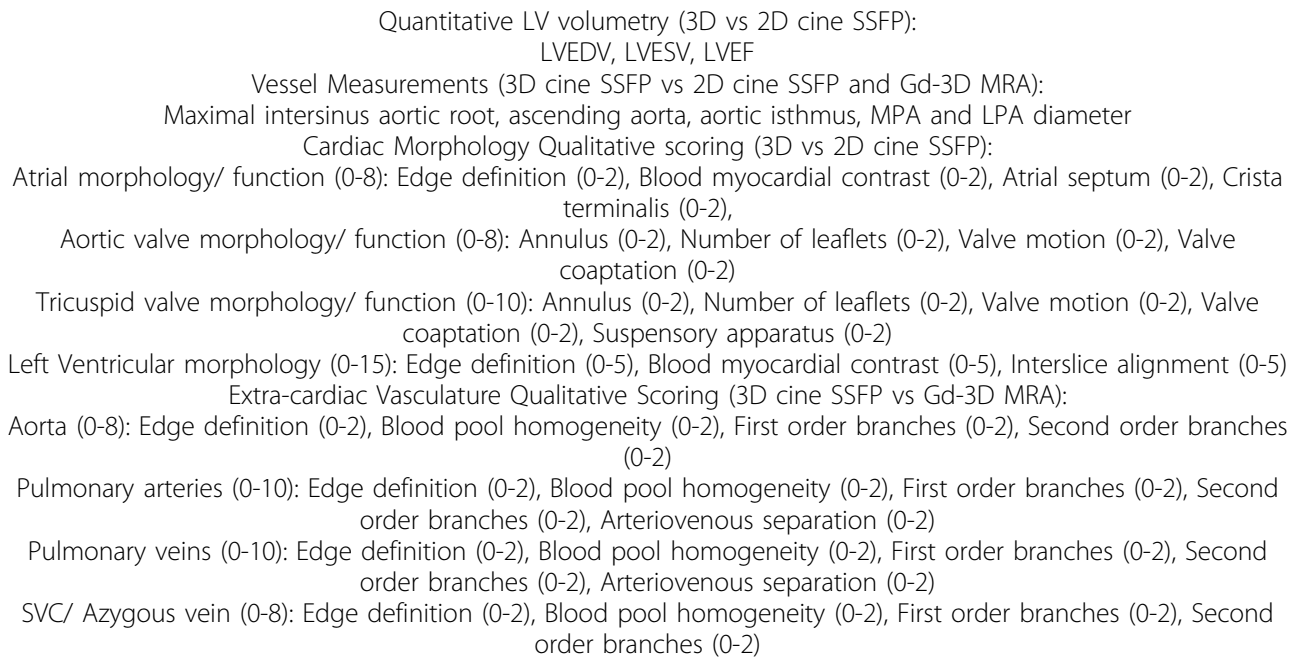 \\
\hline
\end{tabular}

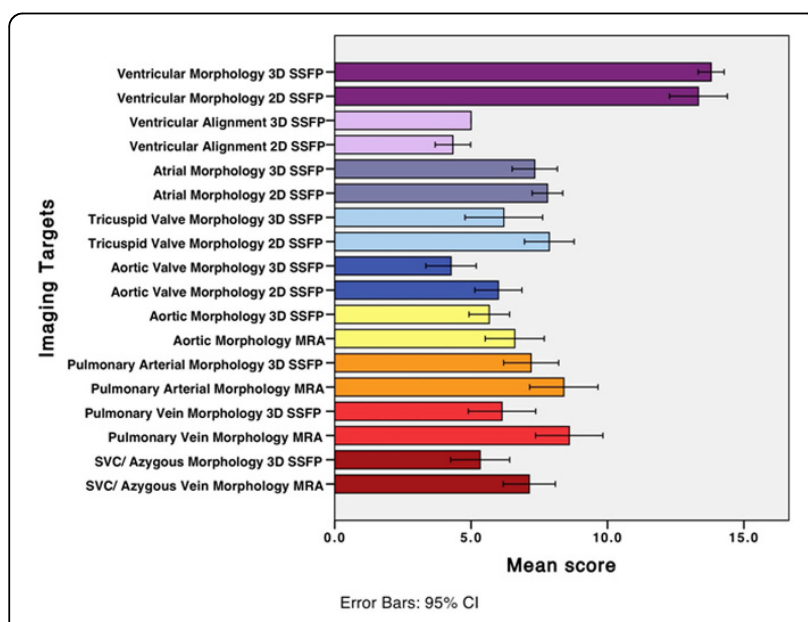

Figure 1 Results comparison of RT-3D SSFP with conventional CMR sequences for morphology and morphology and function in CHD

\section{Funding}

$\mathrm{n} / \mathrm{a}$.

\begin{abstract}
Authors' details
${ }^{1}$ Diagnostic Radiology, American University of Beirut Medical Center, Beirut, Lebanon. 'EB Singleton Department of Pediatric Radiology, Texas Children's Hospital, Houston, TX, USA. ${ }^{3}$ Philips Healthcare, Houston, TX, USA.
\end{abstract}

Published: 3 February 2015
doi:10.1186/1532-429X-17-S1-P29

Cite this article as: Atweh et al:: Comparison of Respiratory-Triggered (RT) 3D cine steady-state free precession cardiac mri with standard 2D cine imaging and magnetic resonance angiography in Congenital Heart Disease (CHD). Journal of Cardiovascular Magnetic Resonance 2015 17(Suppl 1):P29.

\section{Submit your next manuscript to BioMed Central and take full advantage of:}

- Convenient online submission

- Thorough peer review

- No space constraints or color figure charges

- Immediate publication on acceptance

- Inclusion in PubMed, CAS, Scopus and Google Scholar

- Research which is freely available for redistribution
C Biomed Central 